\title{
PAKISTAN SIGN LANGUAGE: COMPUTER VISION ANALYSIS \& RECOMMENDATIONS
}

\author{
BILAL HASSAN $^{1}$, MUHAMMAD SHOAIB FAROOQ ${ }^{1 *}$, ADNAN ABID $^{1}$, NABEEL SABIR KHAN $^{1}$ \\ ${ }^{1}$ Department of Computer Sciences, University of Management \& Technology Lahore, Pakistan \\ Email: \{bilal.hassan, shoaib.farooq, adnan.abid, nabeel.sabir\}@umt.edu.pk
}

\begin{abstract}
Revised November 2016
ABSTRACT. Pakistan sign language (PSL) is one of the sign languages of the world used by the Pakistani deaf community. Unfortunately, PSL has developed over the years without any greater attention from the state institutions. Most of the efforts for the development of PSL are done at the individual or group level, which is mostly impressed by regional or traditional norms. That's why, the learning and teaching PSL is being done in various regions as per their developed version of PSL. A communications barrier occurs when the deaf of two different regions interact with each other as they are trained on their own version of PSL. Another major problem is related to the communication with normal people. The normal people in our society are completely unaware from the signs/gestures of PSL. This communication barrier seizes the basic right of communication between a deaf and a normal. Our contribution in this regard is actually two tiers. First, to the best of our knowledge, we have tried to combine all the efforts made for the development of PSL till date. Second, as a computer vision researcher, we have tried to analyze all the imaging systems developed to aid deaf to deaf and deaf to normal communication of PSL. We are hopeful that this effort will surely help to develop the systems for reducing gap between deaf to deaf, deaf to normal and Pakistan deaf to any other deaf communication.
\end{abstract}

Keywords: PSL; Computer Vision; Gestures; Signs; Deaf

1. Introduction. It has been discussed above that a noticeable amount of deaf community exists in Pakistan. A detail study has been presented based upon statistical data regarding the deaf people in Pakistan [1][2]. About 3.3 million of Pakistanis are suffering from any kinds of disability in which 0.24 million are impaired of hearing which approximates to $7.4 \%$ of overall disables. A very important point is that $55 \%$ of the total disabled lie in the age group from 5 -29 years. Another statistics provide province wise breakdown and facilities for the deaf provided by Government of Pakistan. The report is jointly prepared from statistics which are taken from the Census Report of Pakistan 1998, State of World Refugees and UNHCR Global Report 2002 available at [3]. Along with that various organizations from Pakistan and across from the world made studies about the amount of hearing impaired in Pakistan and the facilities available to them in [4 - 7].

This huge quantity of hearing impaired people demands special attention from the community for minimizing communication gap between them and normal individuals of society. The goal of minimizing communication gap among the deaf and between a deaf and normal man can only be reduced using some sort of signs or gestures. There exist various organizations and research groups in Pakistan which are actively working for development of sign or gestures based languages for Pakistani deaf [8, 9]. As per their opinion, these sign/gesture based languages will help them in several ways like performing day to day activities in a casual manner, helping them in education and reducing the gap between a deaf and normal individual. The unavailability of any standard sign language and special deaf teachers are also the major problems in deaf communication and education in Pakistan as discussed by Bushra et al [10].

Apart from that people have also done lots of efforts to aid the deaf community of Pakistan in various ways. The details of various efforts made are available at [11]. They have observed their behaviors, cultures. Their interests, hobbies and extracurricular activities were also been observed. Their response towards marital life 
has also been the part of study. The community not also tried to aid the deaf in Pakistan but also outside the Pakistan.

It was necessary here to provide a statistical background about the quantity of hearing impaired in Pakistan. We are hopeful that this ground reality will definitely convey a message to the various research communities to come forward and facilitate the hearing impaired. We as people from ICT and particularly from Computer Vision are very much interested in the development of systems which can capture the signs/gestures performed by a deaf and translate them into textual, oral or any other form which is easier to understand. Our goal here is effective communication. Over the years, people from ICT provided various solutions for deaf learning and teaching. The details of these resources are coming in the next sections.

The people from computer vision also took it as an opportunity and developed systems which can capture the sign/gesture, recognize it and transform into an understandable form by the deaf or normal at the other end. They have developed several systems which has ability to recognize signs/gestures performed and achieved very handsome results. The details of the efforts are coming in the next sections.

The ultimate objective of the people from ICT was to facilitate the deaf in day to day communication. An important hazard faced by the ICT community every time was the unavailability of any kind of standard PSL version. We have discussed above that PSL has evolved over the years by the efforts from individuals or groups. We have discussed the development history of PSL in the next sections.

At this point we were in confusion. We decided to collect and analyze all the efforts made for the development of PSL. There were several objectives for this effort. One of the greater objectives was to motivate people for the development of a standardized and accepted by all version of PSL. This process was only possible if we able to bring all the community working on the development of PSL on a single platform. That's why in this effort we targeted the PSL development community as our first audience. We have collected and shared all the development efforts in this research. We are hopeful that this knowledge will definitely convey a message for standardization.

We have divided our work into multiple sections. The section II discusses the evolution of PSL. The section III discusses the aids to deaf from computing community i.e. efforts from ICT community and particularly from computer vision community. The analysis on current state of the art developments and systems has been presented in section IV. Our suggestions and recommendations which can integrate PSL with sign languages of rest of the world in this section is also part of this section. Finally, conclusion of our study in presented in section $\mathrm{V}$.

2. Evolution of PSL. The history of Pakistan sign language is not so rich. It started at individuals/groups levels without any support from the state institutions. To the best of our knowledge, the traceable history of PSL can be started form the initiative taken by Syed Iftikhar Ahmad [12]. It was a PSL dictionary which contains 750 signs of various uses. As it was at individual level, that's why it covered the gestures of Rawalpindi city. However, it was a foundation effort which introduced the gestures/signs for single handed Urdu alphabets.

Another major contribution made by "Anjuman-e-Behbood-e-Samat-e-Atfal" (ABSA) for the development of PSL. It's a Research Group established to document and standardize the PSL. ABSA published several books on sign language available at [12].

The efforts from the state also didn't stopped. A special authority named "National Institute of Special Education" (NISE) for the sate established for the development of manpower which can run special education centers $[12,13]$.

A privately administrated NGO named "Pakistan Association of the Deaf" (PAD) by the ten deaf. They realized the need of a particular sign language accepted by all and for this they published four books as well [14]. Those books covered signs for Urdu and English alphabets, a dictionary of new words and the modified ones along with traffic signs/gestures for deaf drivers. They have also published a book which contains Urdu Grammar for Sign Language and ICT tool for learning PSL.

3. Computing Aid for Deaf. To the best of our knowledge, first ICT assisted learning tool sponsored by United States with the aid amount of US $\$ 28500$ started in 2002 by Sabahat [15]. The project provided lessons for learning and evaluation exercises in the form of $\mathrm{CD}$ and a web portal. The objective of the project was to enhance and facilitate the learning capabilities of deaf in Pakistan.

Along with development of sign language, people have also provided their effort for learning. A very recent advancement is the development of categorical PSL learning resource [16]. The project contains a 
5,000 word PSL Visual searchable database. The dataset is available in form of DVD, mobile app and a book contains 1,000 signs of Urdu, English and four main regional languages spoken in Pakistan.

Computer Vision Based PSL Systems. Boltay Haath was one of the pioneers Pakistan Sign Language Recognition System [17]. Boltay Haath used a computer based gesture recognition system and a data glove for converting signs produced by a deaf into audible speech. Their technique is based upon statistical template matching which receives incoming sign as input and compares it with the stored one in dataset of signs/gestures. They have taken hundred instances against each letter of English and Urdu alphabet. They have found an accuracy level ranging from $70-80 \%$. There are several limitations in their work which impacts a lot like cost of data glove, use of only one data glove which can be used to perform the signs/gestures which are possible with only one hand (However, there are several signs in PSL which requires both hands).

Another approach using data glove but this time the colored one is proposed by Sumaira et al [18]. They have used a fuzzy classifier to recognize the signs/gestures performed by the deaf. Their algorithm uses the angle between finger tip and joint for classification of gesture. Their dataset was based on Urdu alphabets of Pakistan sign language. They have achieved an overall accuracy of $95 \%$ as 35 out of 37 alphabets were recognized correctly. Again, the cost of color data glove, recognition of single hand gestures and static gestures are the limitations of their system.

An inverse system is deployed by Ahmed et al which serves the deaf community in different way [9]. Their system 'PSLIM' reduces the communication gap between the hearing and a hearing impaired person. They listen to the audio and convert it into sign/gesture of PSL. They have used the vocabulary from developed by Zahoor [14]. They have used various built in API's for the development of their system and achieved an overall accuracy of about 78\%. The limited amount of PSL vocabulary and imprecision of speech recognizer and Machine translation were the items to be improved in their system.

Another work for reducing communication gap between the deaf and normal is done by Asif Ali [19]. They have proposed a system which takes input in both forms text and image of sign and convert it into other form. They have performed this for Urdu alphabets of PSL using Haar classifier. They have used a simple RGB camera for this purpose. However, they have not specifically mentioned the size and nature of dataset used for experimentation. The accuracy rate of developed system is also missing.

An image processing based approach for the recognition of Urdu signs/gestures of PSL has been proposed by Khan et al. [20] Deaf as novice can also learn computer programming and software engineering etc. Farooq et al. [21 - 25] propose a comprehensive framework and pedagogically effective subset of C++. They have captured 500 images of 37 letters of Urdu alphabets by using an RGB camera. They have used image processing techniques for skin region filtering. After that they extracted the features of interest by applying discrete wavelet transformation (DWT) and trained a neural network for recognition/classification purpose. To the best of our knowledge, this is the first approach without using any kind of hardware like data glove etc. They have presented their output in textual form. Out of 500 images they have selected 426 for training and 74 for testing and achieved an accuracy of about $86 \%$. The salient feature of their work is that it handles the pose variation of hand as well. However, their work is restricted only for static and single hand gestures.

Another recent work related to recognition of Urdu alphabets of Pakistan sign language is done by Sami et al [2]. There work is another addition regarding recognition of Pakistan sign language using computer vision techniques. They have used cross correlation to find closed match between input image and the image dataset. They have performed their experimentation for the 37 alphabets of Urdu sign language and achieved an accuracy of about $75 \%$. Again the limitations of their work include the recognition of static and single hand gestures.

3. Analysis \& Recommendations. We have tried our best to find out the current efforts accomplished successfully to aid deaf community in Pakistan. We have gone through the web and analyzed the work completed in this particular domain. Based upon our study, we prepared our analysis report in terms of some key findings. We are hopeful that these key points will definitely help the community to move ahead.

The first and the foremost item which has a significant important is the unavailability of PSL version accepted by all. People have done efforts for the development of sign language at individual or group level or somehow at state level. However, most part of their language is impressed by their regional culture and traditional norms etc. This is the basic need which needs to be resolved by joining a single platform. The 
dictionary for PSL should be unique across Pakistan. This kind of effort will surely facilitate the ICT community to aid deaf community in a much better way.

Language is a paradigm which follows some defined grammatical rules. Across the world, all the natural and computer languages follow some particularly defined grammar. That's why, we claim, that a sign language should also follow the grammar rules. Unfortunately, PSL is missing the grammar as it is based upon regional and traditional culture. An effort towards grammar based learning is being done by Pakistan Association of Deaf [14].

After all of the above study, we focused our self on our key domain which is computer vision for deaf. Computer Vision is the process of taking images of the signs/gestures, analyze them and present their description in textual/oral or any other appropriate form. The very first thing in this activity is to get aware the deaf research community about the powers of computer vision techniques. So, a space to work between both of the communities can be established. From this point, we expect both of the communities computer vision and deaf as our audience. In our above presented studies, we have presented points of interests for both of them. We are hopeful that our effort will join both of the communities much closer.

For computer vision community, the unavailability of accepted by all deaf signs/gestures dataset is a big issue. That's why the training and testing of algorithms developed by the computer vision community is a challenge right now. A complete signs/gestures dataset containing multiple body part sign/gesture, static and dynamic as well in an appropriate output form is the need of the time. That's why; a support from the PSL development community at this stage is essential to move ahead.

Current Computer Vision Practices World Wide to Aid Deaf. Throughout the world, the computer vision community is very active. They are utilizing their best efforts to aid deaf. If we think deaf signs/gestures as human skeleton movements, we can easily categorize them in the field of human activity recognition. The Microsoft's 3D sensor Kinect introduces itself as a skeleton capturing device and diverted the mindset of human activity recognition community towards itself. Initially, people used its RGB and 3D information for the recognition of signs/gestures of the deaf languages. Later, Microsoft itself modified Kinect for capturing finger movements as most of the sign/gesture based languages uses fingers mostly for performing signs/gestures. As per our opinion, the use of Kinect sensor for PSL will be a great idea.

The world of deaf has also taken a different direction now. They are not limiting them only in their regional language like PSL etc. They are crossing the borders and doing efforts for the developments of cross lingual or multilingual systems. As per their opinion, these kinds of systems will reduce the communication gap between the deaf of various regions. An effort made in this regard is the development of a system which can facilitate the communication between the deaf from Chinese and United States [26]. The system uses Microsoft Kinect sensor. We also think that we as computer vision community should move one step ahead. We suggest that we should integrate our self in collation with Chinese and US using Kinect translator for sign language. This will definitely be a greater contribution for Pakistani hearing impaired and will reduce the communication barrier for them while communicating across the world in any terms like education, health or sports.

Conclusion. In our work, we have targeted the audience from computer vision and deaf community and tried to join them on a single platform. We have presented statics regarding deaf in Pakistan. We have presented the origins and development history of sign/gesture language in Pakistan. We have presented our suggestions for the development of accepted by all version of PSL. We have collected and analyzed the aid provided by the ICT community particularly from computer vision community for the deaf to best of our knowledge. We have also presented the problems faced by ICT/computer vision community while developing systems to aid the deaf in Pakistan. We have invited PSL development community to join and facilitate the ICT community. We are hopeful, that our effort will bring both of the communities on a single platform. An era of standardization in PSL will be started. A language free of regional and cultural norms but following some defined grammatical rules will emerge. The people from computer vision community will be more comfortable for working. Also the efforts for multilingual and cross lingual communication will continue.

\section{REFERENCES}

[1] Alvi, A. K., Azhar, M. Y. B., Usman, M., Mumtaz, S., Rafiq, S., Rehman, R. U., \& Ahmed, I. (2004). Pakistan sign language recognition using statistical template matching. International Journal of 
Information Technology, 1(1), 1-12.

[2] Sami, M., Ahmed, H., Wahid, A., Siraj, U., Ahmed, F., Shahid, S., \& Ali Shah, S. I. (2014, April). Pose recognition using cross correlation for static images of Urdu sign language. In Robotics and Emerging Allied Technologies in Engineering (iCREATE), 2014 International Conference on (pp. 200-204). IEEE.

[3] Statisitcs, D. (2015). Deaf Statistics. Pakistan Association of Deaf. Retrieved 6 December 2015, from http://Padeaf.org

[4] Waqar, K. (2014). Disability:Situation in Pakistan (1st ed.). Karachi: Right To Education Pakistan. Retrieved from http://www.itacec.org/document/gaw/gaw2014/2.\%20Disability\%20Pages\%202.pdf

[5] Consulting, A. (2012). Disability Evaluation Report (1st ed.). Karachi: Pakistan Poverty Allevation Fund. Retrieved from http://www.ppaf.org.pk/Sector/evaluation\%20report.pdf

[6] United Nations Economic and Social Commission for Asia and the Pacific (ESCAP),. (2013). Expert Group Meeting on Effective Data Generation for the Incheon Strategy Indicators. Retrieved from http://www.unescap.org/events/expert-group-meeting-effective-data-generation-incheon-strategy-indicat ors

[7] Awan, M. (2012). PERSONS WITH DISABILITIES (PWDs) STATISTICS IN PAKISTAN 2012. Islamabad: Agha Gee Printers - Islamabad.

[8] Sulman, D. N., \& Zuberi, S. (2000). Pakistan Sign Language-A Synopsis.Pakistan., June.

[9] Haseeb, A. A., Illyas, A., \& Ilyas, A. Speech Translation into Pakistan Sign.

[10] Akram, B., \& Bashir, R. Special Education and Deaf Children in Pakistan: An Overview. Journal of Elementary Education, 22(2), 33-44.

[11] Tzur, D. (2000). Deaf cultures: Pakistan. Theinterpretersfriend.org. Retrieved 6 December 2015, from http://theinterpretersfriend.org/indj/dcoew/pakistan.html.

[12] Deaf. S. (2014). 'Index of /', Sda.com.pk, . Retrieved 6 December 2015, from http://www.sda.com.pk/.

[13] National Institute of Special Education,. (1991). Pakistan Sign Language Book I and II.

[14] Pakistan Association of the Deaf,. (2014). Deaf in Pakistan. Retrieved 6 December 2015, from http://www.padeaf.org/publication.asp. [Accessed: 04- Sep- 2014].

[15] International Development Research Centre (IDRC),. (2002). Information and Communication Technologies (ICTS) Assisted Learning Tool for the Deaf in Pakistan. Retrieved from http://web.idrc.ca/en/ev-22754-201-1-DO_TOPIC.html

[16] Resources, L. (2015). Family Educational Services Foundation. Family Educational Services Foundation. Retrieved 6 December 2015, from http://www.fesf.org.pk/

[17] Alvi, A. K., Azhar, M. Y. B., Usman, M., Mumtaz, S., Rafiq, S., Rehman, R. U., \& Ahmed, I. (2004). Pakistan sign language recognition using statistical template matching. International Journal of Information Technology, 1(1), 1-12.

[18] Kausar, S., Javed, M. Y., \& Sohail, S. (2008, August). Recognition of gestures in Pakistani sign language using fuzzy classifier. In Proceedings of the 8th conference on Signal processing, computational geometry and artificial vision(pp. 101-105). World Scientific and Engineering Academy and Society (WSEAS).

[19] Ali, S. A. Detection of Urdu Sign Language using Harr Algorithms.

[20] Farooq, M. S., Khan, S. A., Abid, K., Ahmad, F., Naeem, M. A., Shafiq3a, M., \& Abid, A.(2015) Taxonomy And Design Considerations For Comments in Programming Languages: A Quality Perspective. Journal of Quality and Technology Management.

[21] Khan, N. S., Abid, A., Abid, K., Farooq, U., Farooq, M. S., \& Jameel, H. Speak Pakistan: Challenges in Developing Pakistan Sign Language using Information Technology. South Asian Studies, 30, 2.

[22] Farooq, M. S., Khan, S. A., Ahmad, F., Islam, S., \& Abid, A. (2014). An evaluation framework and comparative analysis of the widely used first programming languages. PloS one, 9(2), 02.

[23] Farooq, M. S., Abid, A., Khan, S. A., Naeem, M. A., Farooq, A., Abid, K., \& Shafiq, M. (2012). A Qualitative Framework for Introducing Programming Language at High School. Journal of Quality and Technology Management, 8, 135-151.

[24] Akram, U., Hasan, B., Ullah, S., Bhatti, S., Hassan, F., \& Bashir, J. (2014). A Study on RE Process Models for Offshore Software Development. Journal Of Basic And Applied Scientific Research, 4(4), $114-119$ 
[25] Hassan, B., Farooq, S., Abid, A., Daanial, Y., Sabir, N., \& Naseer, M. et al. (2014). Requirement Engineering Practices in Pakistan Software Industry: Major Problems. Journal Of Applied Environmental And Biological Sciences, 4(7S), 391-397.

[26] Microsoft Research,. (2013). Kinect Sign Language Translator expands communication possibilities. Retrieved from

http://research.microsoft.com/en-us/collaboration/stories/kinect-sign-language-translator.aspx 